\title{
A New Branching Model: Application to Carotid Ultrasonic Data
}

\author{
Alexandre Moreau-Gaudry ${ }^{1}$, Philippe Cinquin ${ }^{1}$ and Jean-Philippe Baguet ${ }^{2}$ \\ 1 TIMC-IMAG, Institut Albert Bonniot, 38706 La Tronche cedex, FRANCE \\ \{Alexandre.Moreau-Gaudry, Philippe.Cinquin\}eimag.fr \\ 2 Department of Internal Medicine and Cardiology, Hôpital Michallon, CHU \\ Grenoble, FRANCE
}

\begin{abstract}
Ultrasonic acquisition, by its simplicity, low cost and non invasiveness, emerged as an essential supplementary examination for cardiovascular diseases. The aim of this study is to demonstrate the feasibility of a global branching artery modelling. A geometrical approach is used for that purpose. A surface, based on a deformable skeleton and envelope, is defined. Including the shape as an a priori knowledge, this surface is fitted with 3D ultrasonic data by an iterative downhill gradient descent. Quality of the fit is illustrated by figures and numerical results. This tool could be used for objective spatial quantification and can be a useful instrument for blood flow modelling.
\end{abstract}

\section{INTRODUCTION}

In industrialized countries, cardiovascular diseases are the most significant mortality causes. In terms of frequency, morbidity and mortality, coronary diseases represent the most important part. Infraclinic atherosclerosis is an abnormality in-between cardiovascular risk factors and athero-thrombotic complications. Its ultrasonic diagnosis on peripherical arteries, particularly carotid, is going to benefit from the introduction of $2.5 \mathrm{D}$ ultrasound ( $2 \mathrm{D}$ ultrasonic data located in $3 \mathrm{D}$ space [4]). Our objective is to initialize a $3 \mathrm{D}$ automatic analysis of the whole artery by fitting a global branching model to the branching of the carotid. This defines an absolute reference system, that can improve the reproductibility of the ultrasonic exploration: tissular modifications can be followed in this reference system. Such a system would be a potentially useful and objective tool to follow disease outcome or to describe therapeutic treatment efficiency.

\section{A NEW BRANCHING MODEL}

In the first approximation, an arterial branching is a volumetric "Y". From this ensues one of the principal modelling difficulties: the shape is not homotopic to a cylinder, a sphere, or a torus. So, we can not use deformable models like $[5,9]$ only defined for one of these different topologies. Furthermore, generalization of these approaches to a branching topology appears as a difficult problem. 
A branching rebuilding was considered in [6] but it doesn't deal with it globally: each branch of the branching artery is rebuilt independently from the two others. The branching heart is then obtained by an interpolation between the three branches. In this context, the a priori knowledge intrinsically contained in the branching is not used for data fitting.

Another approach for rough branching reconstruction was presented in [1] by the use of implicit iso-surfaces generated by skeletons. In this rebuilding, we find again the previously described pitfall, but it could be interesting to establish relations between different parts of skeletons to integrate the a priori knowledge.

Topologically adaptative deformable models are quoted in [8]. These have been shown to be promising in segmenting vascular structures in $2 \mathrm{D}$ and $3 \mathrm{D}$ images and in dealing effectively with branching. But contrary to these last two approaches, we focus on an explicit modeling of branches.

The model that we developed includes the shape as an a priori knowledge. Its presentation is divided in two parts: the first one describes shape building, and the second one presents the classical rigid transformations applied to our model.

\subsection{THE SHAPE}

A shape description is presented figure 1. A surface $M(t, v)$ is a function of two parameters $t$ and $v$. Because a branching appears to be characterized by its upper convexity, we model it by a plane curve, named external skeleton $\mathbf{S}_{\mathbf{e}}$ and parameterized by $t$. To create the surface delimiting the branching, plane closed curves, parameterized by $v$, are built continuously on $S_{e}$ : their mathematical envelope then defines the branching. Each plane containing the plane curve is characterized by only one $t$; coefficients defining the curve are a function of $t$ because of the continuous building.

As regards the look of our plane curve, because we want to be synthetic and realistic, a plane superquadric seems to be a good candidate. Three elements are essential for its definition: the center and the two axis. One axis is defined in direction and norm by the center $C$ of the superquadric and the point $M_{e}$ of $S_{e}$ on which the superquadric is built. Now, if we consider the set of all centers when $t$ is varying, we are naturally driven to the notion of the internal skeleton $\mathbf{S}_{\mathbf{i}}$.

So, the shape is made up of a skeleton and an envelope. This envelope is supported by the skeleton. The skeleton is split in two components, internal and external.

The external skeleton $S_{e}$. To keep the complexity down, $S_{e}$ must have a minimal number of degrees of freedom. We choose a "polynomial" parametric plane curve coming from the cissoïd family [7] according to our modelling concept of the branching convexity and its simple definition by three shape parameters. The coordinates of the current point $M e(t) t \in[-1 . .1]$ are shown in the equation (1). 


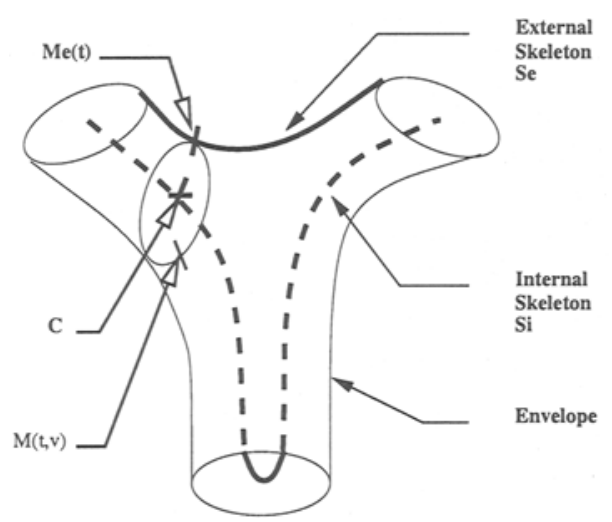

Fig. 1. Description of the shape

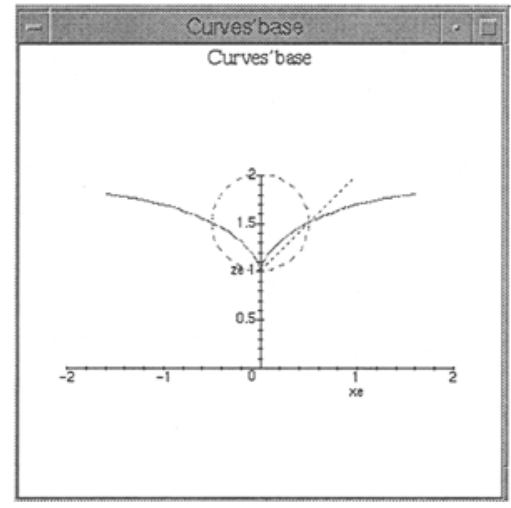

Fig. 2. Plane curves' base

$$
M e(t)=\left(\begin{array}{l}
\frac{a_{1} * t+a_{2} * t^{2}+a_{3} * t^{3}}{1+t^{2}} \\
0 \\
1+\frac{t^{2}}{1+t^{2}}
\end{array}\right)=C+a 1 * M 1(t)+a 2 * M 2(t)+a 3 * M 3(t) .
$$

$M 3(t)$ covers a cissoïd and gives the branching idea. $M 2(t)$, describing a line, creates asymmetry. $M 1(t)$ is on a circle, making round $S_{e}$ (see figure 2). This decomposition shows advantages of this external skeleton parameterization: we are "polynomial" with natural basic deformations. For the same degrees of freedom, to build a model from a parabolic curve, for example, is not so obvious. Figure 4 shows different forms of $S_{e}$ (thin upper curves).

The internal skeleton $S_{i}$. The middle part of $S_{i}$ is defined by a plane curve coming from the cissoïd family (parameterized by $u$ ). Two splines with four control points are added on each side to take information along upper branches into account. $C 1$ continuity is assured. At this development state of the model, it is characterized by 14 parameters: three for $S_{e}$, three for the central part of $S_{i}$, and four parameters for each lateral splines. Figure 3 presents the building. Figure 4 shows different forms of $S_{i}$ (thick lower curves).

Consistency of the skeleton. To guarantee the model definition, we must avoid geometric intersections between $S_{e}$ and $S_{i}$. For this reason, constraints on different shape parameters are imposed: $S_{i}$ is then characterized by five parameters. Figure 3 shows examples of constraints on control points: points at the ends of $S_{i} P_{4 r}$ and $P_{4 l}$ are forced to travel on the half line orthogonal to the speed vector in $M e(1)$ and $M e(-1)$. Points $P_{3 r}$ and $P_{3 l}$ cover segments of horizontal line. $P_{1 r}, P_{1 l}, P_{2 r}, P_{2 l}$ are fixed by $C 1$ continuity. Figure 4 shows different forms of skeleton.

Furthermore, to build a surface parameterized by $t$, we have to establish a one-to-one mapping between $S_{i}$ and $S_{e}$. Let $M_{i}(u)$ be the unique point of $S_{i}$ 


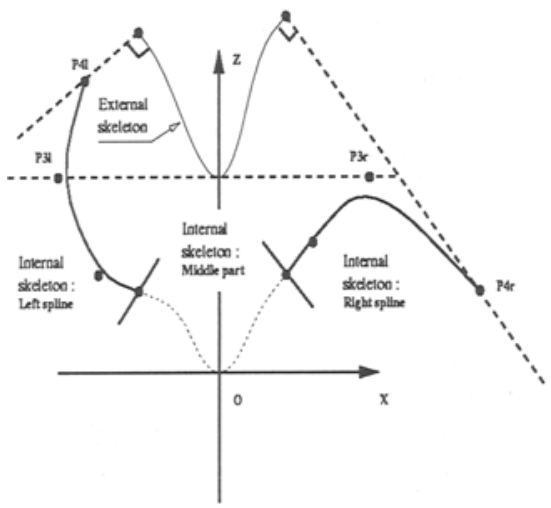

Fig. 3. Internal skeleton building with constraints on control points

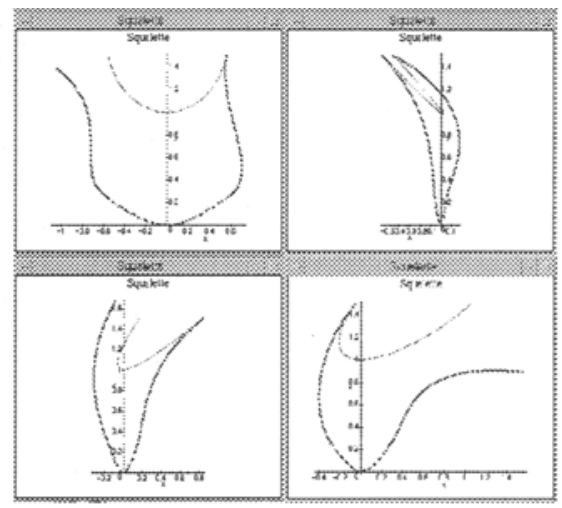

Fig. 4. Example of skeleton for different shape parameters

corresponding to $M_{e}(t)$, one point of $S_{e}$. We propose to define $M_{i}(u)$ by the equation (2) :

$$
\overrightarrow{M_{i}(u) M_{e}(t)} \cdot \frac{d \overrightarrow{M_{e}(t)}}{d t}=0 .
$$

This last equation is cubic in $u$. Thanks to the constraints on parameters, we obtain an explicit solution $u$ of $t$ by the use of CARDAN's formula. Each point of $S_{i}$ is then located by a unique $t$ (see figure 5). An explicit relation between these two curves is a main point for the model efficiency, because no numerical estimations are needed for describing exactly the surface.

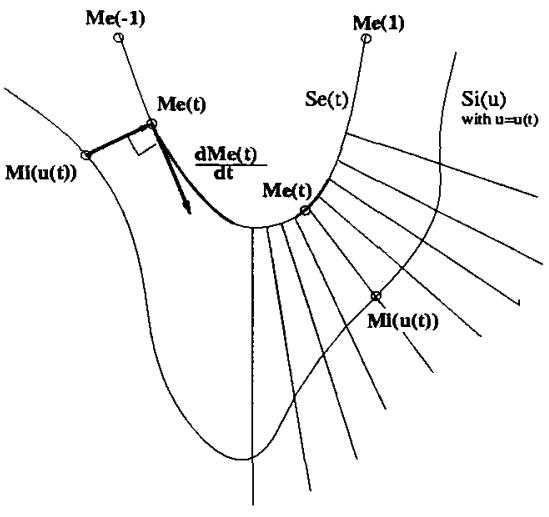

Fig. 5. Illustration of the one-to-one mapping between $S_{e}$ and $S_{i}$

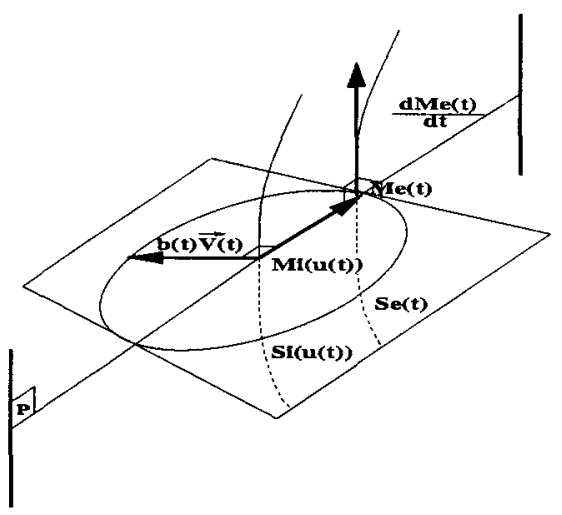

Fig. 6. Representation of the minor axis 
The envelope. The final surface is obtained by a continuous juxtaposition of $2 \mathrm{D}$ superquadrics [3]. Each superquadric $i$ is bound to the external skeleton at point $M_{e}\left(t_{i}\right)$. Its center is the image $M_{i}\left(u\left(t_{i}\right)\right)$ by the bijection defined in (2). Thus, the branching surface is defined by the following $3 \mathrm{D}$ vector:

$$
\begin{aligned}
& M(t, v)=\vec{A}(t, v)+\vec{B}(t, v) . \\
& \vec{A}(t, v)=\overrightarrow{M_{i}(u(t)) M_{e}(t)} * \cos (v)^{\epsilon(t)} . \\
& \vec{B}(t, v)=b(t) * \overrightarrow{V(t)} * \sin (v)^{\epsilon(t)} .
\end{aligned}
$$

$\overrightarrow{V(t)}$ is the unit vector normal to the plane $\mathcal{P}$ in $M_{i}(u(t)) . \mathcal{P}$ is defined by the equation (4). An illustration is shown in the figure 6 .

$$
\mathcal{P}:\left(M_{i}(u(t)), \overrightarrow{M_{i}(u(t)) M_{e}(t)}, \frac{d \overrightarrow{M_{e}(t)}}{d t}\right) .
$$

$b(t)$ represents the "minor" axis norm of the 2D superquadric: it is defined for $t \in[-1,1]$ by two splines with four control points, with a $C 1$ continuity in $t=0$. Six constraint parameters are necessary to characterize it.

$\epsilon(t)$ is a function defined for $t \in[-1,1]$ with values in $] 0,1]$. This function allows deformations of $2 \mathrm{D}$ superquadrics from an ellipse to a rectangle: a better fit can then be reached in the central region of the artery. Its definition using three parameters $\alpha, \beta, \gamma$ is the following:

$$
\begin{gathered}
\epsilon(t)=\alpha+(1-\alpha) * \frac{\frac{\delta^{2}(t)}{1+\delta^{2}(t)}}{\frac{\beta^{2}}{1+\beta^{2}}} . \\
\delta(t)=\beta *(t+\gamma) .
\end{gathered}
$$

Finally, we build a branching shape with 17 parameters (shape parameters). Each parameter value has been specifically restricted according to geometric model properties to maintain a meaning shape. Examples of surfaces are presented in the figure 7 .

\subsection{RIGID TRANSFORMATIONS}

The rigid transformations applied to our model are classically defined by $7 \mathrm{pa}-$ rameters (spatial parameters): three Euler angles $\psi, \theta, \varphi$, three translation parameters $T_{x}, T_{y}, T_{z}$ and one size factor $S$.

\section{MINIMIZATION METHOD}

Let $N_{d}$ data points $M_{i} i=1 . . N_{d}$ extracted from ultrasonic data (see section 4). An energy $\mathcal{E}$ is defined in (6) as the euclidean distance of data points to the surface. 


$$
\mathcal{E}=\sum_{i=1}^{N_{d}}\left\|\overrightarrow{M_{i} \mathcal{P}_{M_{i}}\left(t_{i}, v_{i}, X\right)}\right\|^{2} .
$$

It is a function of 24 parameters $X_{j} j_{j=1 . .24}: 17$ shape parameters and 7 spatial parameters. $\mathcal{P}_{M_{i}}\left(t_{i}, v_{i}, X\right)$ represents the projection of $M_{i}$ on the surface, $t_{i}, v_{i}$ its parameters. An explicit derivative of the cost function according to each parameter $X_{j}$ is computed by Maple software (Maple V Release 4). We use a Fletcher-Reeves-Polak-Ribiere minimization [10]. During this iterative descent method, we respect the constraints on each parameter by a simple projection according to the descent direction. To decrease time consuming, we do not estimate the energy function during a descent step. All spatial and shape parameters are adjusted simultaneously, so most of the given data points lie close to the model's surface. Initial spatial position is defined manually by selection of the three branching ends. Initial shape parameters are fixed to neutral position according to the definition field. Because of the non convexity of the cost function, and the non convexity of the constraints domain, this minimization drives us to the nearest local minima.

\section{DATA COLLECTION}

Data are obtained with collaboration of the Department of Internal Medicine and Cardiology at the Grenoble University Hospital. Echographic images are acquired by a HP SONOS (Hewlett-Packard, Santa Clara, California, USA) ultrasonic system. They are stored on an optical disk and computed on a workstation (HP 715/100). A set of $43662 \mathrm{D}$ points are generated by a semiautomatic segmentation step.

During the medical exploration, the ultrasonic system is connected to an optical localizer (Optotrak Northern Digital Inc.). To collect 2.5D data [4], a six degrees-of-freedom optical tracking device attached to the ultrasound probe is used. The optical tracking system works with infrared diodes put on the probe which are then located by three fixed CCD cameras (Optotrak Northern Digital Inc.). Position and orientation of the echographic planes are thus obtained during all the exploration. Thanks to a temporal synchronization between echographic and spatial data, 3D spatial coordinates for each previous $2 \mathrm{D}$ points are computed.

\section{RESULTS}

Thanks to the a priori knowledge contained in the model, the initialization of the minimization process is easy. Figure $7 \mathrm{c}$ shows distance from data to surface resulting from the minimization process. Compared to the figure $7 \mathrm{a}$, one can clearly notice a visual improvment with regard to the data fitting. Numerically, with the previously described data collection, the rms for the cost function decreases from 1.17 to 0.56 . Because of the great number of data, the time limiting element of the minimization process is the downhill direction evaluation. 


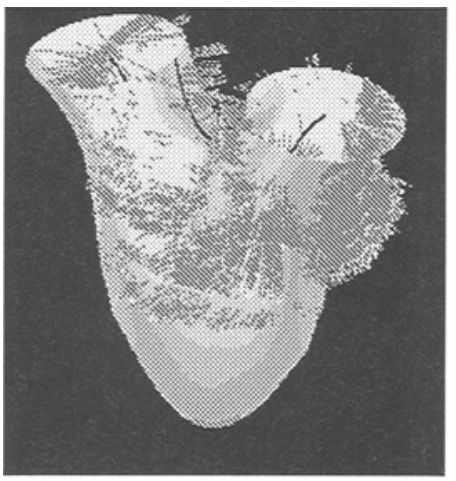

(a)

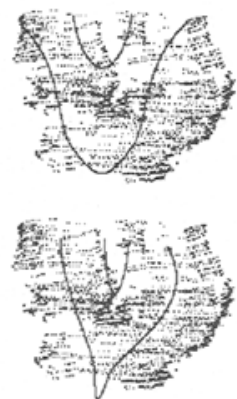

(b)

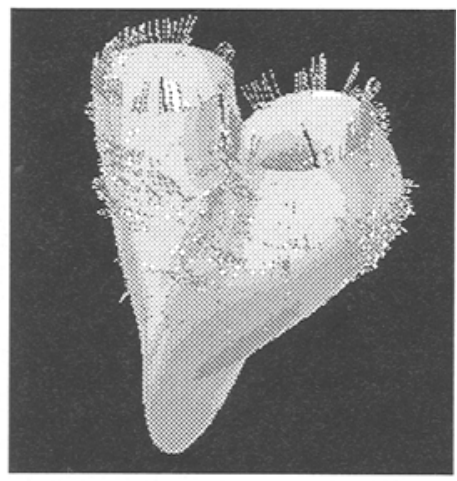

(c)

Fig.7. (a) shows initial surface, distance (gray lines) from data (white points) to the initial surface and parts of the skeleton (black lines). (b) represents data (black points) and deformations of the skeleton (black lines): the top part of the figure shows the initial skeleton; the bottom part, the final skeleton resulting from the minimization process. (c) illustrates final surface, distance (gray lines) from data (white points) to the final surface, and parts of the skeleton (black lines).

\section{DISCUSSION AND CONCLUSION}

\subsection{ADVANTAGES AND PITFALLS}

In medical ultrasonic investigation, image analysis is in progress, but only few methods are able to describe automatically the division of one artery in two branches $[6,2]$ : the first one uses an interpolation of explicit generalized cylinders; the second one uses an implicit approach. To our knowledge, no explicit deformable branching model incorporating the shape as an a priori information had been developed. The aim of this geometric model is to demonstrate the feasibility of modelling the branching artery globally. This region of interest is fitted in one step by 24 parameters. Our tool takes into account the angle between the two derived arterial branches. A potential asymmetry between the branches is included by our model, which confirms its deformation capacity.

One of the pitfalls of our model is the relatively high number of parameters. Pertinence of model's parameters must be tested in the future. In the same time, parameters constraints may be reviewed to impose identifiability of the model.

\subsection{PERSPECTIVES}

The top part of the figure $7 \mathrm{~b}$ shows data and initial skeleton; the bottom, the same data, the skeleton resulting from the minimization process and an unappropriatness between the size of each branch and the deepest point of the branching convexity. This remark could explain the relatively bad value of the residual error. The minimization result seems overevaluated. It could be easily improved by adding another longitudinal scale parameter for the external skeleton. 
Our data collection corresponds to a cross sectional study. We need some longitudinal information to test the reproducibility and the parameters sensibility of our model. This tool is promising. It could be used for all the branching vessels acquired by ultrasonic system (femoral, renal, popliteal divisions). It is known that ultrasonic slices are very noisy. Investigations by CT scan or MRN could be interesting. The use of our model could be extended, particularly to cerebral, coronary or hepatic branching vessels.

Our geometric model will be the first step to an accurate quantification of this artery part. It will be a measurement tool to evaluate new medical therapeutics tested to slow down atherosclerosis evolution. It could be an element in medical decision making, in order to optimize the cost/benefit equation.

At last, in physiological medical research, our model which creates a virtual branching surface from 3D real data could be included in hemodynamic vascular model to test the local repercussion of turbulence on the atherosclerosis outcome.

Acknowledgments: This work was supported by Hewlett-Packard and Medasys Digital Systems.

\section{References}

1. E. Bittar, N. Tsingos, and M-P. Gascuel. Automatic Reconstruction of Unstructured 3D Data: Combining a Medial Axis and Implicit Surfaces. Computer Graphics forum (Eurographics'95), 14(3):457-468, August 1995.

2. D.Attali E.Ferley, M.P.Cani-Gascuel. Skeletal Reconstruction of Branching Shapes. The Eurographics Association, 1997.

3. F.Solina and R.Bajcsy. Recovery of parametric models from range images : The case for superquadrics with global deformations. IEEE Transactions on Pattern Analysis and Machine Intelligence, 12(2):131-147, February 1990.

4. G.Champleboux and D.Henry. Parametric two-dimensional b-spline, representation of vein and artery from $2.5 \mathrm{~d}$ echography used to aid virtual echography. CVRMed-MRCAS'97-LNCS, pages 285-294, 1997.

5. Lawrence H.Staib and James S.Duncan. Model-based deformable surface finding for medical images. IEEE Transactions on Medical Imaging, 15:720-731, October 1996.

6. V.Juhan B.Nazarian K.Malkani R.Bulot J.M.Bartoli and J.Sequeira. Geometrical modelling of abdominal aortic aneurysms. CVRED-MRCAS, pages 243-252, 1996.

7. VEB Bibliographisches Intitut Leipzig, editor. Mathematics at a glance. 1975.

8. T. McInerney and D. Terzopoulos. Deformable models in medical image analysis: a survey. Medical Image Analysis, 1(2):91-108, 1996.

9. S. Benayoun C.Nastar N.Ayache. Dense non-rigid motion estimation in sequence of 3d images using differential constraints. CVRMed, 1995.

10. W.H.Press W.T.Vetterling S.A.Teukolsky and B.P.Flannery. Numerical recipies in $c$ : Second edition. 